\title{
Anti-persistence in the global temperature anomaly field
}

\author{
L. M. V. Carvalho ${ }^{1,3}$, A. A. Tsonis ${ }^{2}$, C. Jones ${ }^{3}$, H. R. Rocha ${ }^{1}$, and P. S. Polito ${ }^{4}$ \\ ${ }^{1}$ Dept. of Atmospheric Sciences, University of São Paulo, Brazil \\ ${ }^{2}$ Dept. of Mathematical Sciences, Atmospheric Sciences Group, University of Wisconsin, Milwaukee, USA \\ ${ }^{3}$ Institute for Computational Earth System Sciences, University of California, Santa Barbara, USA \\ ${ }^{4}$ Dept. of Physical Oceanography, University of São Paulo, Brazil
}

Received: 8 February 2007 - Revised: 24 October 2007 - Accepted: 24 October 2007 - Published: 26 November 2007

\begin{abstract}
In this study, low-frequency variations in temperature anomaly are investigated by mapping temperature anomaly records onto random walks. We show evidence that global overturns in trends of temperature anomalies occur on decadal time-scales as part of the natural variability of the climate system. Paleoclimatic summer records in Europe and New-Zealand provide further support for these findings as they indicate that anti-persistence of temperature anomalies on decadal time-scale have occurred in the last $226 \mathrm{yrs}$. Atmospheric processes in the subtropics and mid-latitudes of the SH and interactions with the Southern Oceans seem to play an important role to moderate global variations of temperature on decadal time-scales.
\end{abstract}

\section{Introduction}

The Earth's average surface temperature has increased by $0.6 \pm 0.2^{\circ} \mathrm{C}$ since the late 19 th century with significant global impacts (IPCC, 2007). The dramatic heat waves in the Northern Hemisphere (Berniston, 2004; Karl et al., 1995; Karl and Knight, 1997; Meehl and Tebaldi, 2004), extensive collapses of ice shelves over the Artic (Serreze et al., 2003) and Antarctic (Shepherd et al., 2003), recent hurricane activity (Emanuel, 2005) and other major weather events are viewed as a direct result of this warming, which is attributed partly to natural variability of the climate system and partly to human activity. However, uncertainties about the details of the natural variability as well as the response of the climate system to the rapid increase of greenhouse gases still remain (IPCC, 2007). Understanding natural variability of the climate system is crucial to predict non-linear climate forcings and abrupt changes possibly due to anthropogenic activity (Alley et al., 2003). The objective of this study is to im-

Correspondence to: L. M. V. Carvalho

(leila@model.iag.usp.br) prove our understanding of climate variability by providing new insights about the spatiotemporal variability of temperature anomalies.

Here, low-frequency variations in temperature fluctuations on distinct time-scales are investigated by mapping temperature anomaly records onto random walks. Based on this approach, statistics of temperature fluctuations have been successfully applied to demonstrate the importance of El Niño in moderating changes in global temperature (Tsonis et al., 1998; Tsonis et al., 2003). Motivated by this previous study, mapping onto a random walk is used here to describe lowfrequency variations in the sequence of anomalous events over time in a given region and ultimately understand temporal variations and spatial organization of global temperature fluctuations.

This paper is organized as follows: Sect. 2 discusses the data and the rationale for the application of mapping onto a random walk to interpret low-frequency variations of global temperature fluctuations. Section 3 investigates the scaling properties of these random walks to investigate the role of low-frequency variations in the atmosphere in moderating global temperature fluctuations. Section 4 discusses persistence and anti-persistence in trends of temperature fluctuations on decadal time-scales. Section 5 presents an analysis of paleoclimatic data, which confirms this anti-persistence on decadal time-scales in the last 250 years. Section 6 summarizes the main results and conclusions of this study.

\section{Data and methods}

Global daily temperature anomalies are examined in the present climate (1948-2005) using NCEP/NCAR reanalysis with $2.5^{\circ}$ latitude/longitude resolution (Kalnay et al., 1996) and over the past $226 \mathrm{yrs}(1750-1975)$ using reconstructed summer temperature over Europe (Briffa et al., 1988) and New Zealand (Xiong and Palmer 2000). Long-term linear

Published by Copernicus Publications on behalf of the European Geosciences Union and the American Geophysical Union. 

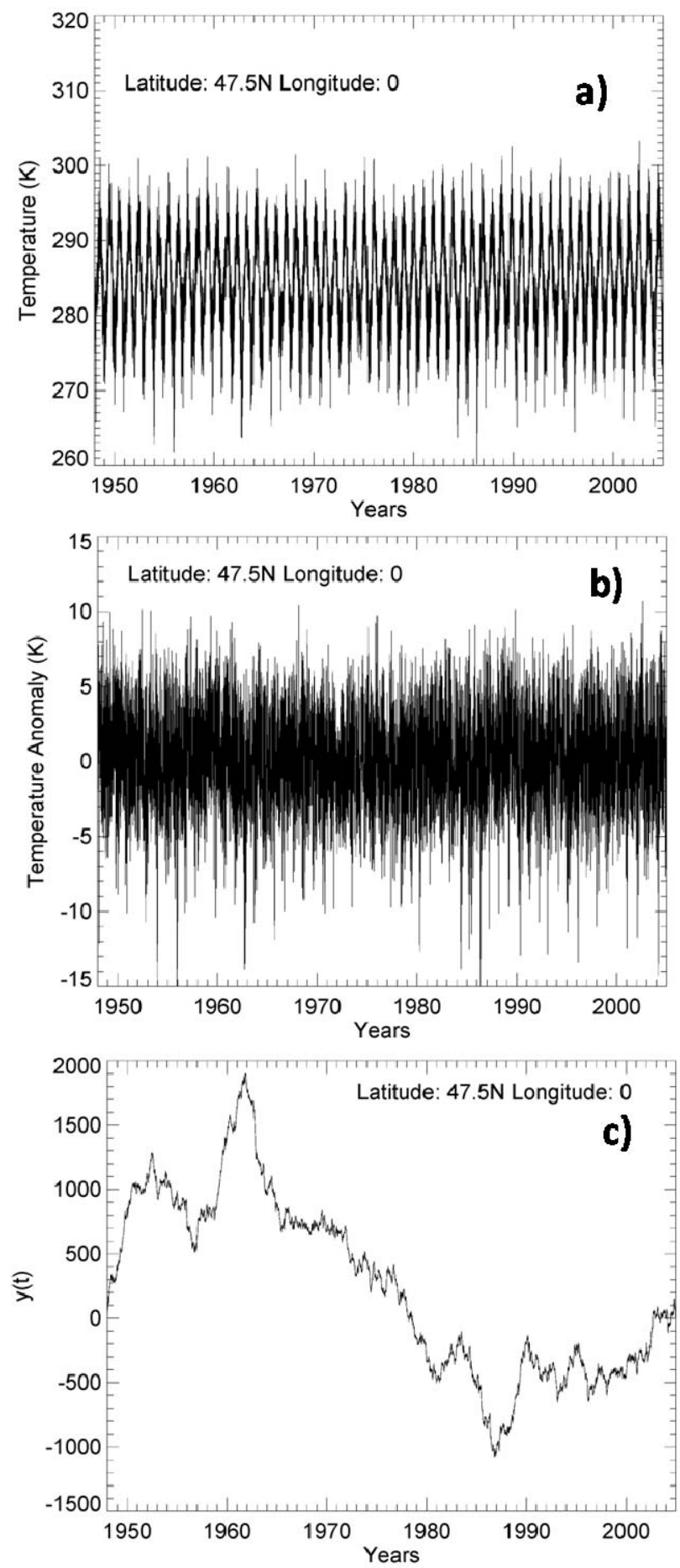

Fig. 1. (a) Example of temperature time series observed at $47.5^{\circ} \mathrm{N}$ and $0^{\circ}$; (b) temperature anomalies $T^{\prime}$ obtained after removing the linear trend and the first and second harmonics of the average daily temperatures for the period 1948-2005; (c) running sum $y(t)$ obtained from temperature anomalies in (b) (see Eq. 1) for the same period. trends are removed from daily temperatures at $2 \mathrm{~m}(\mathrm{~T} 2 \mathrm{~m})$ in the present climate. The annual and, in some regions also the semi-annual cycle, dominate the spectral variance of daily temperatures (e.g. Wilks, 1995). In this study, temperature anomalies $T^{\prime}$ are obtained by removing both cycles, defined as the first and second harmonics of the average daily temperatures for the period 1948-2005. All other oscillations present in the period are maintained. For example, significant power exists in the 4-7 years band corresponding to ENSO. Such features, however, are broadband features and do not represent periodic signals; they are the result of nonlinear dynamics (e.g., Eccles and Tziperman, 2004). As such they should not be removed from the records. Figure 1 shows an example of the raw temperature signal at a grid point (Fig. 1a) and resulting $T^{\prime}$ signal after the long term trend and the annual and semi-annual cycles are removed (Fig. 1b). As Fig. 2 shows, the resulting distributions of $T^{\prime}$ are Gaussian, which is expected for temperature anomalies.

The global spatiotemporal variations of $T^{\prime}$ are interpreted within the framework of random-walks. According to this approach (Tsonis, 1992; Tsonis et al., 2003), the corresponding $T^{\prime}$ signal at a given location is mapped onto a walk by calculating a net displacement $y(i)$ defined by the running sum:

$y(i)=\sum_{j=1}^{i} T^{\prime}(j) \quad i=1,21170$

This running sum describes the sequence of anomalous events over time in a given region and is influenced by the magnitude and persistence of the fluctuations; these in turn depend on complex relationships with the climate system (Tsonis, 1992, Mann et al., 1998). Figure 1c illustrates the running sum (Eq. 1) computed for temperature anomalies shown in Fig. 1b. Mapping onto a random walk is an efficient approach to represent important low-frequency variations in the regime of temperature fluctuations (Tsonis et al., 2003). If climate forcings in a period $\Delta t$ are such that positive (negative) temperature anomalies are more frequent than negative (positive) anomalies, the corresponding running sum exhibits a positive (negative) slope in that time interval. Therefore, time variations in the slope of the running sum are important indications of regime changes in temperature fluctuations.

An appropriate statistical quantity used to characterize the walk is the root mean square fluctuation about the average displacement (Montroll and Shlesinger, 1984; Peng et al., 1992; Buldyrev et al., 1995; Tsonis et al., 2003):

$F(t)=\left[\left\langle[\Delta y(t)]^{2}\right\rangle-\langle[\Delta y(t)]\rangle^{2}\right]^{1 / 2}$

where $\Delta y(t)=y(i+t)-y(i)$, and the symbols $(<>)$ indicate average over all positions $i$ of the walk. Operationally, this is equivalent to the following steps: (1) consider a caliper of distance (time scale) $t=1$, (2) start from the beginning position $(i=1)$ and calculate $\Delta y(1)$ for $i=1$, (3) move to the 
second position $(i=2)$ and calculate $\Delta y(1)$ for $i=2$ and so on, (4) average over all positions the quantities in Eq. (2) to obtain $F(t)$ for $t=1$, (5) repeat for other time scales. Note that in Eq. (2) $F^{2}(t)$ is nothing else than the variance of the increments $\Delta y(t)$.

For a time series $x(i)$, whose values are Gaussian with zero mean and unit variance (white noise), the spectral density scales according to $S_{x}(f) \sim f^{-\beta}$ with $\beta=0$ (flat spectrum). A motion defined by the running sum $y(i)=\sum_{j=1}^{i} x(j)$ is called Brownian motion and its spectral density scales according to $S_{y}(f) \sim f^{-\beta}$ with $\beta=2$. Thus the spectral exponent for the summed time series is +2 larger than the non-summed time series (Malamud and Turcotte, 1999). Note that a Brownian motion has Gaussian increments $\Delta y(t)$. It is well known from theory that the fluctuations $F(t)$ of a Brownian motion scale according to $F(t) \sim t^{H}$ with $H=0.5$. This implies that $2 H+1=\beta$. We can generalize this for any process exhibiting a spectrum that scales according to $S(f) \sim f^{-\beta}, 1 \leq \beta \leq 3$ (such processes are called self-affine processes; in this case it follows that $0 \leq H \leq 1)$. In other words, if $x(i)$ has a characteristic exponent $\beta$ then the running sum (integral) of $x(i)$, $y(i)$, is characterized by a power-spectral exponent $\beta+2$, and if the increments $\Delta y(t)$ are Gaussian with zero mean and a variance that scales according to $F(t) \sim t^{H}(0 \leq H \leq 1)$, then $H$ is related to $\beta$ via the relationship $2 H+1=\beta$ (Peitgen and Saupe, 1988; Malamud and Turcotte, 1999). The exponent $H$ (which is also called the Hausdorff exponent and often denoted as $H_{a}$ ) characterizes the self-affinity of $y(i)$. Note that motions with $H \neq 0.5$ are called fractional Brownian motions (fBm).

It can be shown that $H$ is related to the Hurst exponent, $H_{u}$, (Hurst, 1951; Hurst et al., 1965). The Hurst exponent of a time series varies from zero to one. If it is equal to 0.5 it indicates that the time series is a series of uncorrelated values. If it is greater than 0.5 the time series exhibits persistence, meaning that chances are that a present tendency in the data will continue in the future. If it is less than 0.5 the time series exhibits anti-persistence, meaning that chances are that a present tendency in the data will be reversed in the future. A Gaussian white noise is a sequence of uncorrelated values and therefore it has a $H_{u}=0.5$ and $\beta=0$. This would imply that $2 H_{u}-1=\beta$. The running sum of this white noise has $\beta=2$ and as we discussed above is related to the Hausdorff exponent according to $2 H+1=\beta$. It follows that the Hausdorff exponent of the running sum of $x(i)$ (estimated from the r.m.s. analysis on $y(i))$ is the Hurst exponent of $x(i)$. For example, given an signal $x(i)$, then if we use its running sum $y(i)$ and Eq. (2) and find that $H=0.7$, this will indicate that the corresponding $\beta$ for $y(i)$ is equal to 2.4. Then the corresponding $\beta$ for $x(i)$ is equal to $2.4-2=0.4$, which gives $H_{u}=0.7$, which in turn indicates persistence in our original signal $x(i)$. This relation is expressed as: $H_{u}(\beta-2)=H(\beta)$ (Malamud and Turcotte, 1999). This has been noted earlier by Mandelbrot (1983) where it is stated that as long as the running sum is Gaussian with zero mean and a variance that scales as $t^{2 H}$ (which implies that $F(t) \sim t^{H}$ ), then the Hausdorff exponent can account for the Hurst exponent. Thus, provided that the Hausdorff exponent $H$ is estimated from the variance of the running sum $y(i)$, the following conditions apply to the original signal $x(i)$ (Feder, 1988; Malamud and Turcotte, 1999):

- If $H<0.5$, then $x(i)$ exhibits anti-persistence

- If $H=0.5$, then $x(i)$ is a sequence of uncorrelated values (3)

- If $0.5<H<1$, then $x(i)$ exhibits persistence

The above discussion and relations indicate that both spectral analysis on $x(i)\left[T^{\prime}(i)\right.$ in our case] and fluctuation analysis on $y(i)$ provide the same information. The advantage of using the running sum approach is that it provides a more efficient way to estimate $H$ than spectral analysis does for $\beta$. Direct estimation of $\beta$ is difficult because noise inherent to the spectral density of $T^{\prime}(i)$ masks the presence of scaling regimes. On the other hand $F(t)$ is a much smoother function of $t$, and $H$ is obtained more precisely. The reason is that the mean root mean square fluctuation is related to the autocorrelation function $C(t)=<x(i) x(i+t)>-<x(i)>^{2}$ according to the relation $F^{2}(t)=\sum_{i=1}^{t} \sum_{j=1}^{t} C(j-i)$ (Stanley, 1971). Because $F$ is related to $C$ through a double sum, fluctuations in $F$ are substantially reduced compared to fluctuation in $C$ (which relates to spectral density).

These above concepts reflect the existence of scale invariance, a property inherent to many nonlinear processes in nature (Tsonis, 1992). Therefore, persistence is used here to indicate the tendency for existing temperature fluctuations regime to persist. These relationships are used here to examine low-frequency variations in global temperature.

\section{Time-correlation scaling properties}

Tsonis et al. (1998) applied the mapping onto a walk approach to the monthly global temperature record (18651998). They showed that the global temperature data exhibit two distinct scaling regimes; one consistent with a power law with $H=0.65$ for $t<20$ months and the other with a power law with $H=0.4$ for $t>20$ months. The interpretation is that processes of time scales less than 20 months sustain a tendency toward an initial trend (whether positive or negative) and processes of time-scales greater than 20 months tend to reverse the past trend. They also conclude that this change in behavior defines an important characteristic time scale in global climate. Here we extend this type of analysis to investigate the spatial variation of scaling properties of temperature fluctuations. 


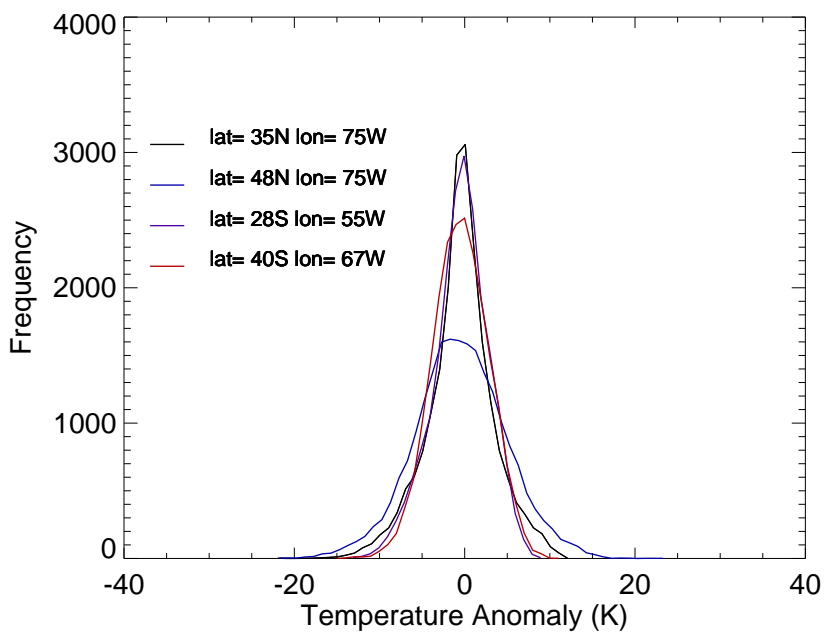

Fig. 2. Frequency distribution of daily temperature anomaly $\left(T^{\prime}\right)$ for selected grid points (1948-2005). Latitude and longitude of each grid point are indicated inside the figure.

In order to examine this issue, we look at variations in the power-law ( $H$ coefficient) in pre-defined scales. This interval of scales yields robust statistics for the determination of $H$, considering the length of data. The scales we investigate correspond to the following time-intervals: $2-15$ days, $20-90$ days, 100-200 days, 2.0-4.0 years, 4.5-6.0 years, and 6.57.0 years. The choice of these ranges is based on the fact that they are known to explain a large fraction of spectral variance of temperature and circulation over the globe between 2 days -7 years (Peixoto and Oort, 1992; Madden and Julian, 1994). The separation in scales does not necessarily imply that we expect a break in scaling properties on these particular scales. Figure 3 exemplifies the $\log (F(t))$ vs. $\log (t)$ plot and respective changes in scaling properties on different time-scales. For this particular grid-point, located at the equator and longitude $100^{\circ} \mathrm{W}, H<0.50$ is observed for timescales longer than two years.

The spatial variability of $H$ for the different time-scales is shown in Fig. 4. On synoptic time-scales (2-15 days) (Fig. 4a), $H$ coefficient is above 0.5 over all regions of the planet which indicates a universal persistence of temperature fluctuations on that time-scale. $H$ is higher over tropical Pacific and Atlantic $(H>0.90)$. The subtropics and extratropics, particularly over the storm track of the Southern Hemisphere and eastern coast of the United States, Southern Australia and southern South America, are associated with relatively lower values of $H$, between 0.6 and 0.7. Cold fronts, cyclones and anti-cyclones are some of the known phenomena affecting temperature on the synoptic time-scales and they occur in nature essentially to equilibrate differences in temperature between tropics and extratropics (e.g. Holton, 2004). Nevertheless, although changes in temperature are certainly observed before and after a passage of a frontal system, these changes do not reverse the long-term trends in temperature anomalies.

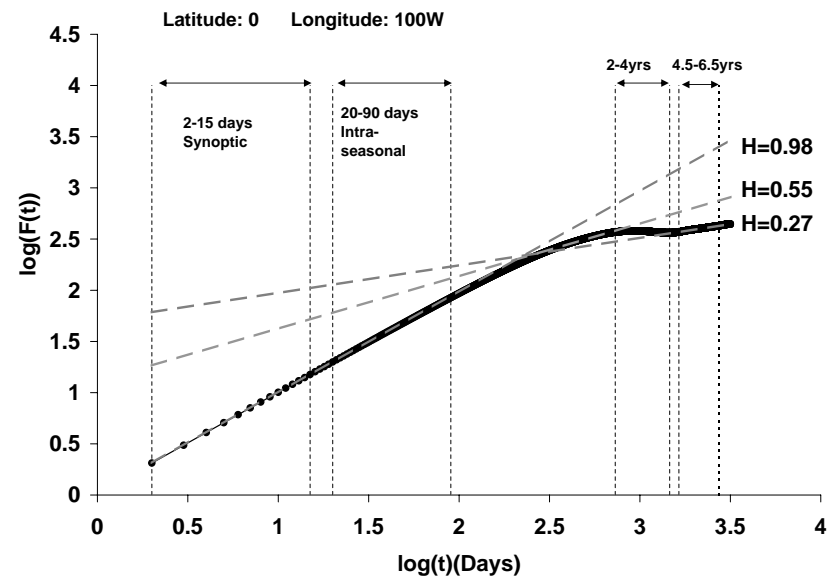

Fig. 3. Example of a $\log (F(t))$ vs. $\log (t)$ plot (dark symbols) for a grid point located at latitude 0 and longitude $100^{\circ} \mathrm{W}$ (1948-2005) and time-scale ranging from 2 days -8 years. The scaling coefficient $H$ observed over distinct time-scales is also indicated (long dashed gray lines).

On intraseasonal time-scales (20-90 days) (Fig. 4b), the universal tendency for persistence continues albeit with lower values $(0.55<H<0.65)$ over continental areas. The Madden-Julian oscillation (Madden and Julian, 1994) is one of the most powerful tropical phenomenon on intraseasonal time-scales that modulates cloudiness, precipitation and circulation over the tropics, with impacts on the subtropics and extratropics of both hemispheres (e.g, Lau and Waliser, 2005, and references therein). In addition, important variations on intraseasonal time-scales have been observed in temperature (Przybylak, 2002) and sea ice (Baba et al., 2006) records in high latitudes. However, the physical reasons for distinct scaling characteristics and, therefore, $H$ over the continents and oceans are not obvious but suggest different responses of temperature fluctuations on intraseasonal time-scales. Difference in the spectrum of maritime and continental stations at high frequencies $\left(f>\right.$ month $\left.^{-1}\right)$ have been also identified in Pelletier (1997).

The 100-200 days time-scale (Fig. 4c) shows spatial characteristics that are similar to intraseasonal (20-90 days) timescales, in particular low $H$ values over the continents. Nevertheless, $H$ decreases in most regions and now approaches to 0.50 , which indicates that for this time-scale future and past trends are likely uncorrelated. In some few areas over the extratropics of the Northern Hemisphere $H$ is $<0.50$. In addition, changes in $H$ also occur over the southern oceans on this scale.

Finally, we focus our attention on scaling properties on interannual to intra-decadal time scales $(2.0-7.0$ years) (Fig. $4 \mathrm{~d}-\mathrm{f}$ ). The general observation now is that as the time scale increases, the tendency for $H$ is to become less than 0.5 almost everywhere on the globe. Arguably, these time scales correspond to the time scale of ENSO, one of the most 

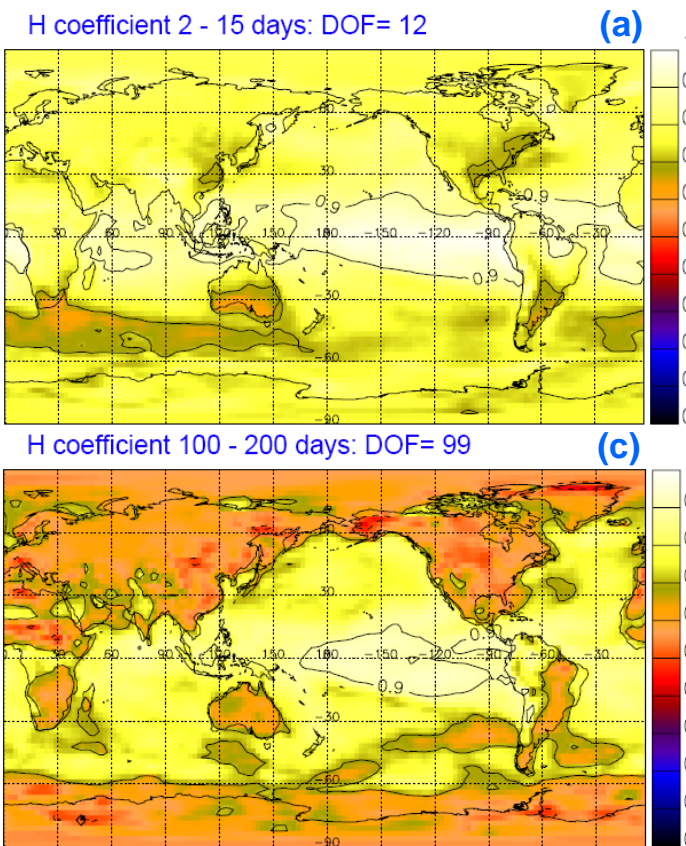

H coefficient $4.5-6$ yrs: DOF $=546$

(e)

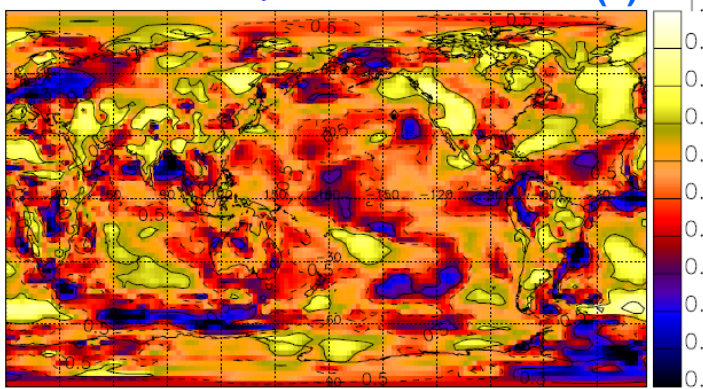

H coefficient 20 - 90 days: DOF $=69$

(b)

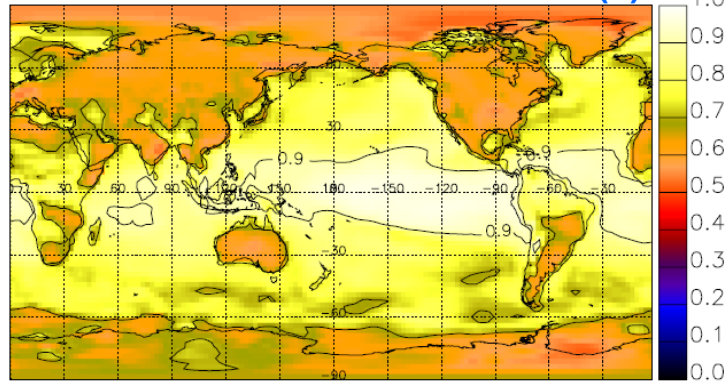

$\mathrm{H}$ coefficient $2-4$ yrs: DOF $=729$

(d)

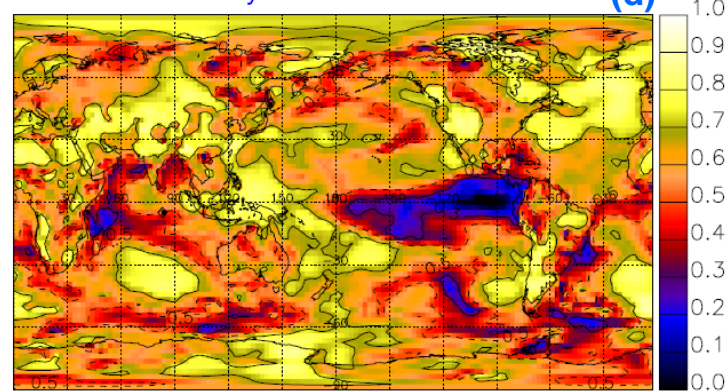

$\mathrm{H}$ coefficient $6.5-7 \mathrm{yrs}$ : DOF $=137$

(f)

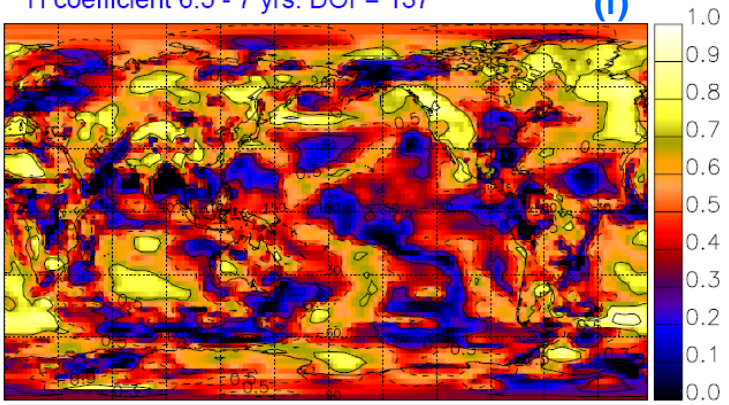

Fig. 4. The scaling coefficient $H$ obtained in distinct time-scales: (a) 2-15 days, (b) 20-90 days, (c) 100-200 days, (d) 2.0-4.0 years, (e) 4.5-6 years and (f) 6.5-7 years. Degrees of freedom are indicated at the top of each panel. Dashed (Solid) lines identify $H<0.5$ ( $H>0.5$ ).

important features of our climate system. This observation supports the hypothesis postulated by Tsonis et al. (2003) that El Niño is a mechanism that reverses previous positive trends of temperature anomalies. In addition to the above variance analysis we present the distributions of the increments $\Delta y(t)$ for various time scales $t$. The distributions are indeed Gaussian with vanishing (zero) mean (Fig. 5). This, together with the power law scaling, satisfies the other condition entering the interpretation of persistence and antipersistence discussed at the end of Sect. 2.

We need to stress here some of the strengths and limitations of our analysis and results. First we emphasize that correlation coefficients of the regression of $\log (F(t))$ vs. $\log (t)$ in the various ranges are very high (above 0.99) and the standard error of the regression coefficient $S_{H}$ are very small for all scales. Figure 6 shows the spatial variability of $S_{H}$ for time scales 2.0-4.0 years (Fig. 6a) and 4.5-6.0 years (Fig. 6b). To emphasize regions with large errors, all grid points with $S_{H}>0.025$ are shaded. Clearly, a very small fraction of grid points shows $S_{H}>0.025$, and no one is observed with errors above 0.05 . For the 6.0-7.5 time scale all grid points show $S_{H}<0.015$ (not shown). However, there is no argument that the fitting is over small ranges (e.g. Maraun et al., 2004). This raises the question of how significant is the reported scaling and thus the proposed anti-persistence. While tests for scaling in cases of a single scaling regime have been proposed (Tsonis and Elsner, 1995), similar tests for multiple scaling regimes do not exist. The reason we trust our results is that if the reported scaling was not significant, then given the small ranges one would expect $H$ to be a rather random variable independent of the time scales considered. This is not what we observe in Fig. 4. What we observe is a coherent structure of persistence for short time scales becoming systematically anti-persistence for longer time scales with features such as the well documented effect of El Niño on temperature tendencies. 


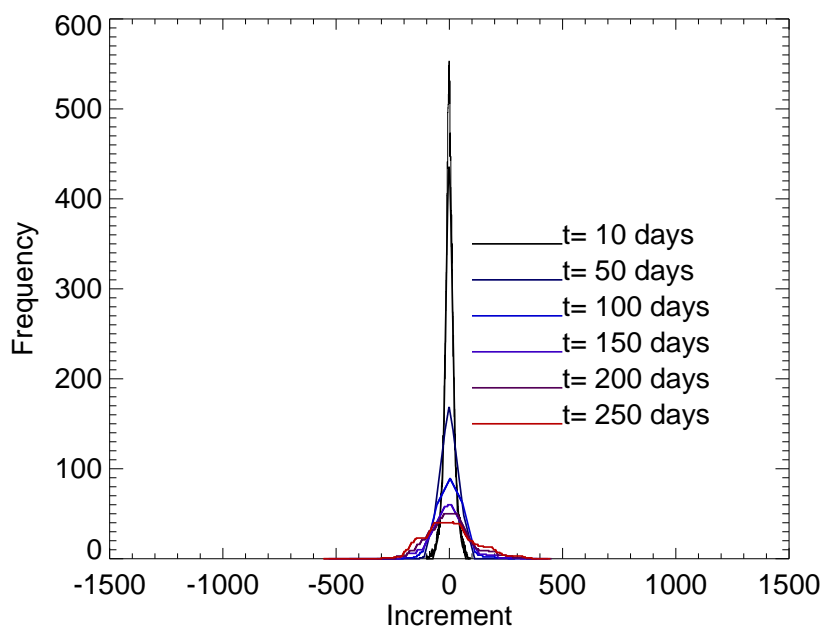

Fig. 5. Example of frequency distributions of the increments $\Delta y(t)=y(i+t)-y(i)$ for time scales $t$ varying from 10 to 250 days. Period of the analysis: 1948-2005.
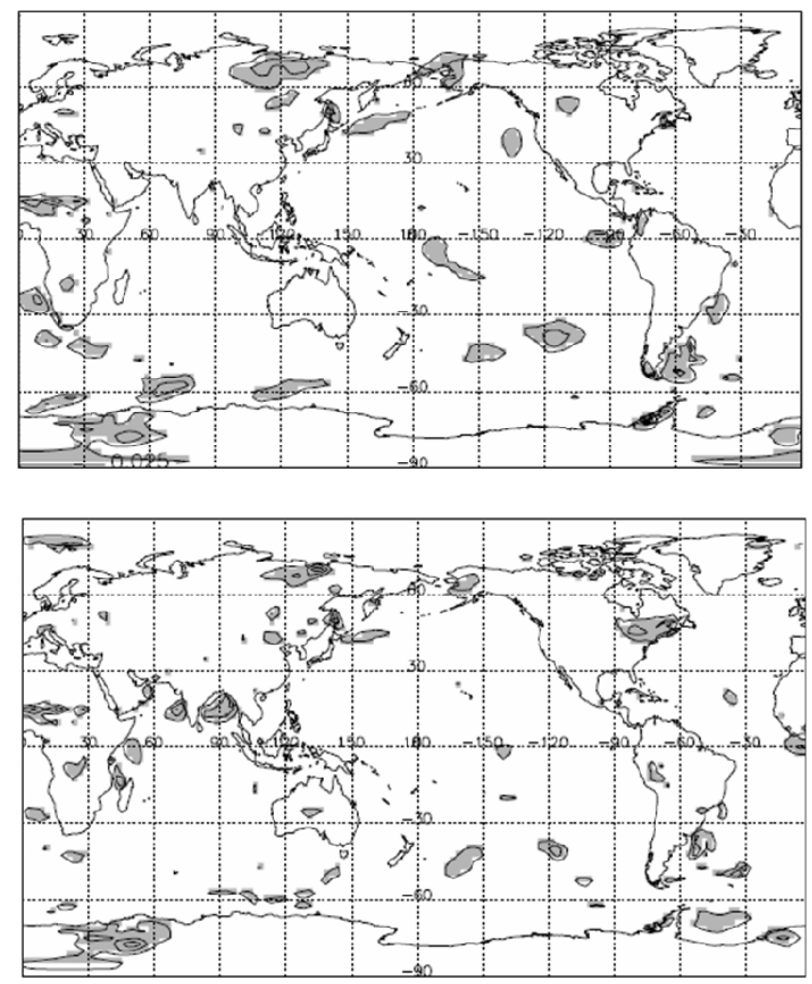

Fig. 6. Regression coefficient standard error $S_{H}$ for timescales 2.0-4.0 years (top) and 4.5-6.0 years (bottom). Values of $S_{H}>0.025$ are shaded. First contour 0.025, contour interval 0.025 .

To offer further support to this tendency (and to account for possible effects due to spatial correlations in the temperature field) we randomly selected 1000 grid points (from a total of over 10368) and generated for each grid point a surrogate Markov process having the same length and lag-1 au-

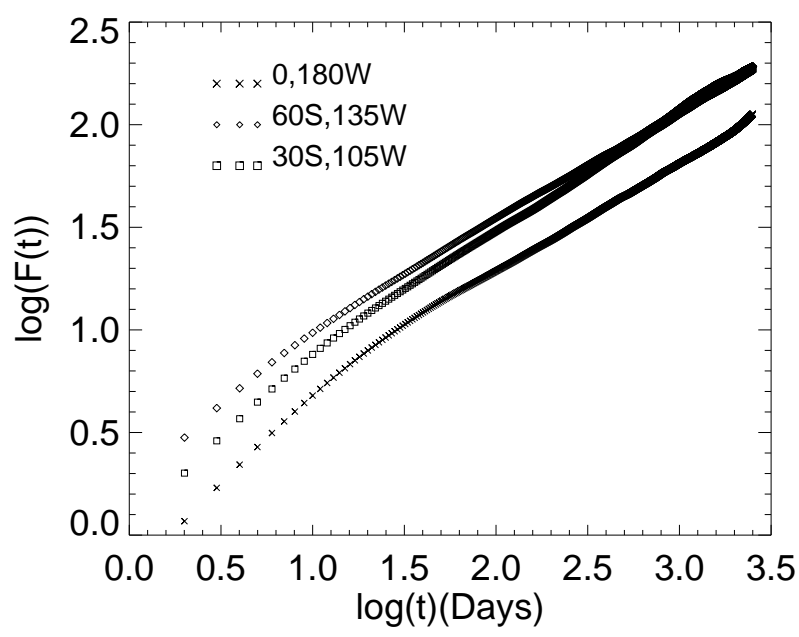

Fig. 7. Examples of a $\log (F(t))$ vs. $\log (t)$ plot for surrogate Markov processes having the same length (1948-2005) and lag-1 autocorrelation as the corresponding temperature anomaly record $T^{\prime}$.

tocorrelation as its temperature anomaly record $T^{\prime}$. We then repeated the analysis and calculated $F(t)$ for each synthetic time series. Figure 7 shows a few examples of the scaling in this case. Now, we do not observe multiple scaling regimes and as expected (Stanley, 1971) the asymptotic behavior beyond the local (in time) correlations is $F(t) \sim t^{0.5}$. We then considered a number of ranges used in Fig. 4 and estimated the corresponding $H$ in those intervals. Figure 8 shows the distribution of $H$ from the surrogate data (solid line) and the actual data (broken line) for four different ranges in time scale. The distributions from the actual data project the general picture in Fig. 4 discussed above: for very short time scales we have complete persistence, which as the time scale becomes longer gives way to anti-persistence. The distributions from the surrogate data, however, tell a different story. Independent of the time scale the distributions are centered at about 0.5 . While the variance increases (possibly due to poorer statistics at longer time scales), there is no tendency whatsoever away from the expected (on the average) value of 0.5 . These significant differences between the distributions of the actual data and surrogates indicate that our results are not likely to arise because of spatial correlations in the data or length of scaling regimes.

Pelletier (1997) has used spectral analysis on temperature records (i.e. on $x(i)$ ) directly and estimated the exponent $\beta$. The overall behavior of the spectra over the range of time scales analyzed in our work appears to be red (i.e. $S_{x}(f) \sim f^{-\beta}$ with $\beta>0$ ). This implies persistence. As we mentioned above the spectral density function is very noisy. As such, while overall behaviors may be identified, anti-persistence over smaller ranges of scales (such as those used in our work) may be masked. For example, in his Fig. 3 one may identify in the range of $\sim 2-10$ years anti-persistence. However, the presence of noise makes this 

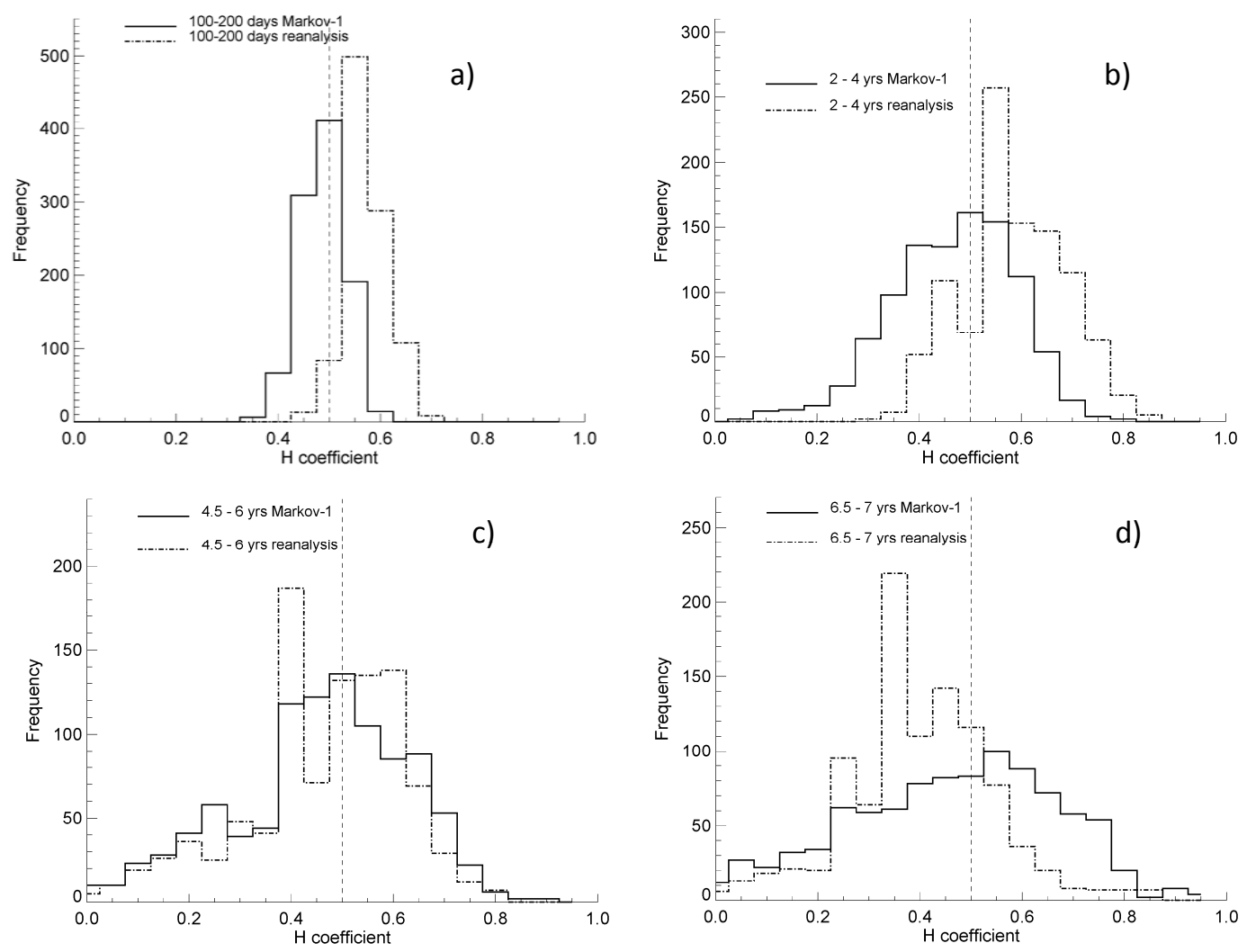

Fig. 8. Frequency distribution of $H$ from 1000 surrogate Markov data (solid line) and 1000 randomly selected actual data (broken line) for four different ranges in time-scale: (a) 100-200 days, (b) 2.0-4.0 years, (c) 4.5-6.0 years and (d) 6.5-7.0 years. The dotted vertical line identifies $H=0.50$. The distributions from the actual data indicate that for very short time scales persistence dominates; on the other hand as the time scale becomes longer anti-persistence becomes more dominating. However, the distributions from the surrogate are centered at about 0.5 for all time scales, as expected from theory.

assessment difficult. Our approach results in much smoother functions and such regimes can be identified more adequately.

In the literature, the sort of analysis performed here is often done by the alternative method Detrended Fluctuation Analysis (DFA) (Koscienly-Bunde et al., 1998). We find no significant differences between DFA and the method we used here. Therefore, while for very short time scales processes in the climate system tend to promote an existing trend, for longer time scales processes are activated to reverse an existing trend, consistent with the results obtained for the global temperature record (Tsonis et al., 1998). Thus, the conclusions reached by using the global temperature record are not just the result of some averaging procedure but reflect the properties of the small scale fluctuations in the temperature field. Due to insufficient statistics in estimating $F(t)$ at longer time scales, we cannot extend this analysis any further in time, but a different approach (see next section) indicates that this anti-persistence may hold on longer time scales as well.

\section{Anti-persistence on long time-scales}

Examination of the produced running sums reveals that significant reversals in the slope of the running sum have occurred on decadal time-scale in large areas over the globe during 1948-2005. Some examples are shown over selected regions in the extratropics of the Northern (Fig. 9a) and Southern (Fig. 9b) Hemispheres. Decadal variations in the running sum are clearly observed. In addition, interannual to intra-decadal variations are also evident, particularly for the Northern Hemisphere (Fig. 9a). The spatial coherence of the running sums is also obvious from the similar temporal variations among neighbor grid points (Fig. 9a, b). No such tendencies were observed in the surrogate data. This almost symmetrical decadal variability of the random walks over regions in the Northern (Fig. 9a) and Southern (Fig. 9b) Hemispheres suggests that the anti-persistence of temperature fluctuations indicated in the analysis above, may extend on decadal time-scales.

In order to investigate this issue we proceed as follows. Over finite time scales, a running sum may exhibit local trends. Therefore, the total running sum length (RSL), 


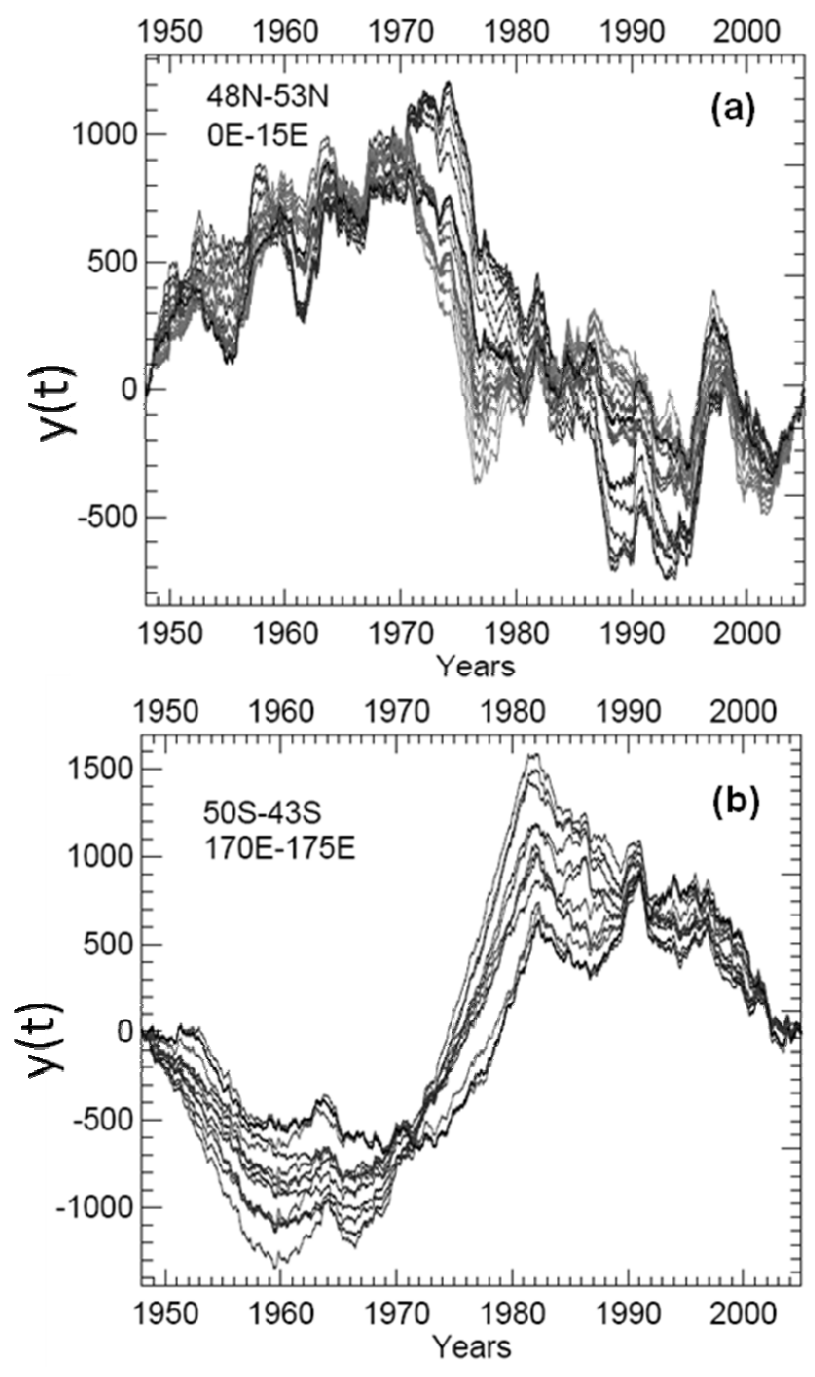

Fig. 9. Running sum (Eq. 1) computed for daily temperature anomalies (1948-2005) for locations in (a) Northern Europe (48$53^{\circ} \mathrm{N}, 0^{\circ} \mathrm{E}-15^{\circ} \mathrm{E}$ ) and (b) South Pacific around New Zealand (40$\left.55^{\circ} \mathrm{S}, 170-178^{\circ} \mathrm{E}\right)$. Each displacement is obtained for a distinct grid point inside the indicated spatial domain.

defined as $\sum y(i)$ over a given period of years is a useful quantity which may yield further evidence of the antipersistence in temperature anomalies on long time-scales. Consequently, random walks were arbitrarily computed in two non-overlapping periods: 1948-1975 (Fig. 10a) and 1976-2005 (Fig. 10b).

Interestingly, areas with high RSL magnitude (positive and negative) switch sign from 1948-1975 to 1976-2005 in a coherent and consistent way with maxima in Fig. 10a becoming minima in Fig. 10b and vice-versa. We observe a general tendency in the first period (Fig. 10a) for positive RSL values in the northern hemisphere and Antarctica (region 1) and a general tendency for negative RSL values in oceans of the southern hemisphere (region 2). This is an indication that
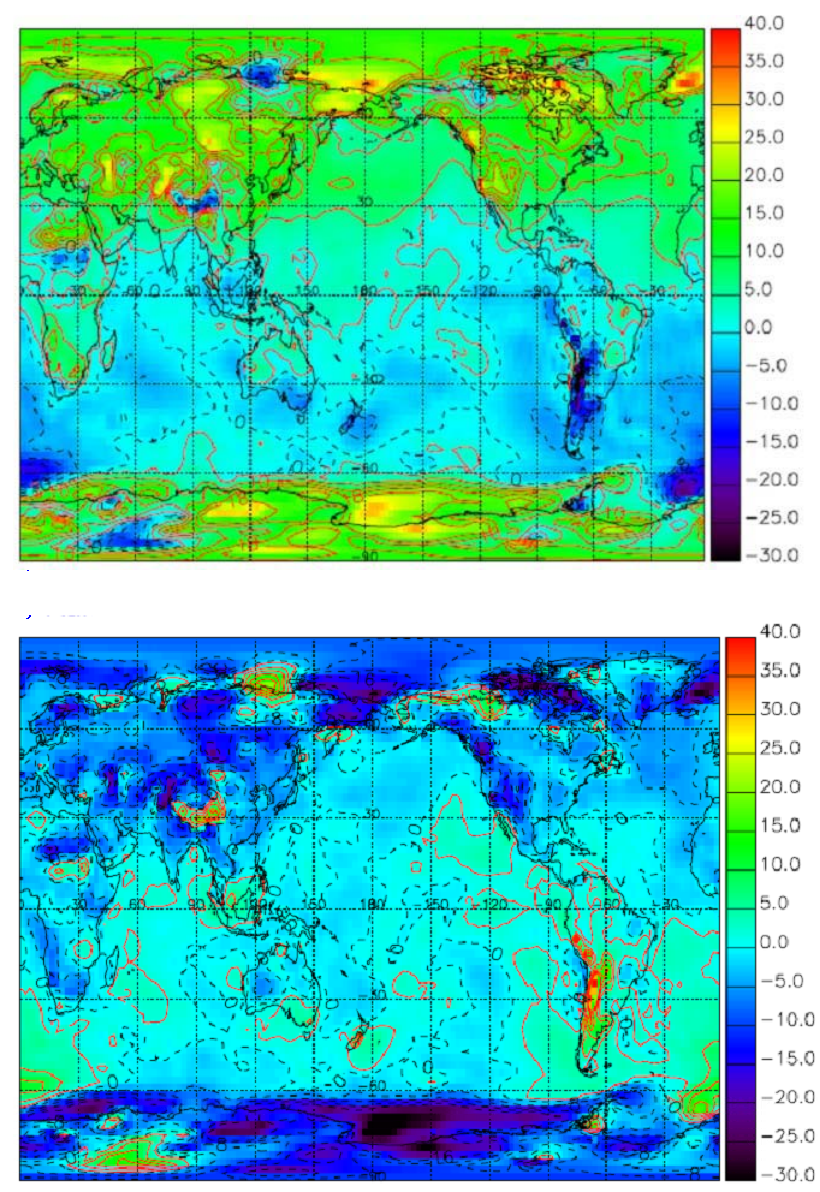

Fig. 10. Running sum length (RSL) for 1948-1975 (top) and 19762006 (bottom). Solid (dashed) lines enclose regions with positive (negative) RSL. Units are $10^{6} \mathrm{~K}$. Note that maxima in Fig. 10 (top) become minima in Fig. 10 (bottom) and vice-versa.

the regime of temperature anomalies in region 1 was such that the frequency and/or intensity of positive temperature anomalies during 1948-1975 preserved the positive trend in the running sum, whereas in region 2 the trends in the running sum were mostly dominated by negative temperature anomalies.

This is an interesting observation but what is remarkable is the reversal observed in Fig. 10b. Now the RSL is negative in region 1 and positive in region 2 . The reversal appears to be universal; it is occurring at almost all grid points and is consistent with the general tendency for anti-persistence observed in Fig. 4 as the time scale increases. This reversal suggests that anti-persistence in temperature fluctuations extends to time scales longer than those of ENSO. This will indicate that decadal time-scales processes are also important in regulating temperature variations. 


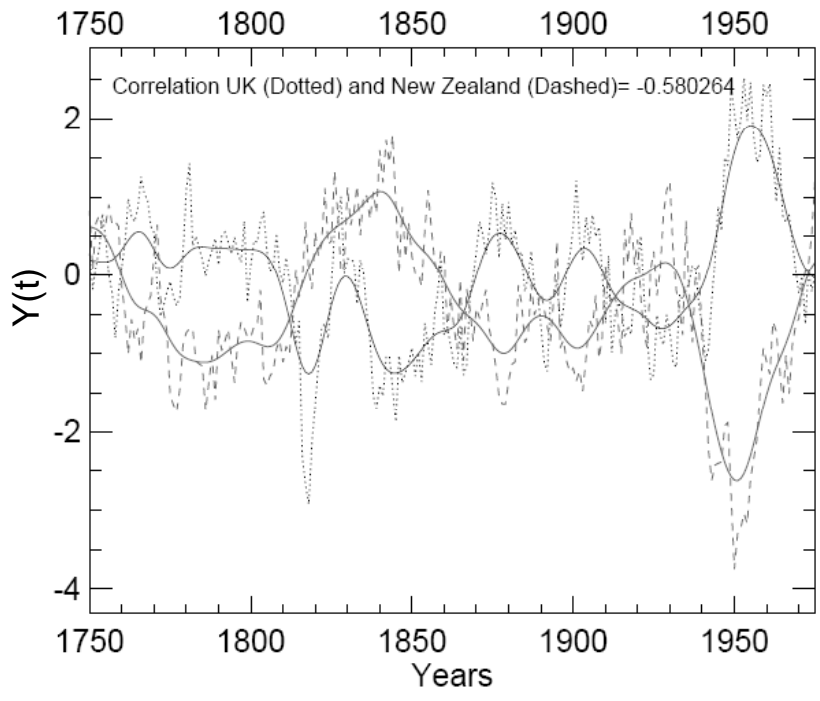

Fig. 11. Running sum (Eq. 1) generated from summer reconstructed temperature anomalies in New Zealand (dashed line) and United Kingdom (dotted line) during 1750-1975. The correlation between these two curves is equal -0.58 and is statistically significant at 5\% significance level. The number of degrees of freedom $\left(N_{\mathrm{dof}}\right)$ is equal to 15 based on the relationship $N_{\mathrm{dof}}=N\left(1-\rho_{1 \mathrm{NZ}} \rho_{1 \mathrm{UK}}\right) /\left(1+\rho_{1 \mathrm{NZ}} \rho_{1 \mathrm{UK}}\right)$, where $N$ is the number of seasons and $\rho_{1 \mathrm{NZ}}\left(\rho_{1 \mathrm{UK}}\right)$ is the lag-1 auto-correlation function for New Zealand (United Kingdom) records. Solid lines are 5 years moving average (200 passes) to highlight low-frequency variability of temperature regimes in New Zealand and United Kingdom.

\section{Anti-persistence in paleoclimatic records}

The suggested anti-persistence on decadal time scales is next examined using paleoclimate data. Fortunately, temperature records exist for Europe (European Gridded and Regional summer reconstruction) (Briffa et al., 1988) and New Zealand (Xiong and Palmer, 2000). These two regions are particularly important for this study because as RSL in Northern Europe is positive, around New Zealand is negative and vice-versa (Figs. 9 and 10). Temperature variations over Europe (EU) were reconstructed for a 6-month summer season (April-September) for the period 1750-1975 using a network of maximum latewood (Briffa et al., 1988). The New Zealand (NZ) data consist of average summer (FebruaryMarch) temperature derived from eleven NZ cedar ring width chronologies (Xiong and Palmer, 2000).

Temperature anomalies in both datasets were obtained with respect to the 1951-1970 period. Trends were removed and the running sums of summer temperature fluctuations were computed according to Eq. (1). Figure 11 shows the two running sums (dotted lines). The correlation coefficient between these two running sums is -0.58 and is statistically significant at the 5\% significance level. The solid lines are 5year moving average and show correlation equals to -0.78 . These results indicate that the remarkable anti-persistence

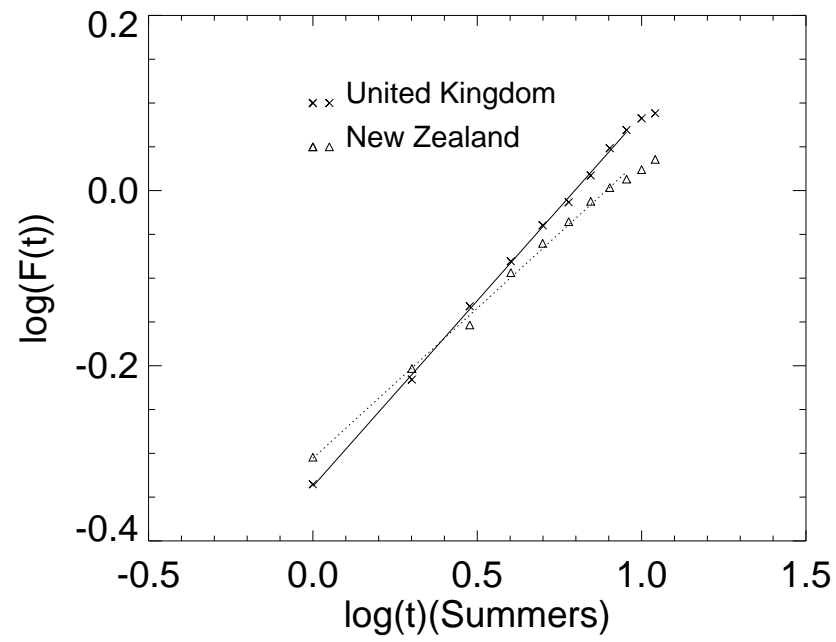

Fig. 12. The $\log (F(t))$ vs. $\log (t)$ function from the net displacements in Fig. 11. United Kingdom (solid line), New Zealand (dotted line).

on decadal time-scale and the anti-correlation between New Zealand and UK datasets have been present in the last 2.5 centuries. The reconstructed datasets are summer averages in both hemispheres and, consequently, the anti-correlation is not an artifact of computing random walks over distinct seasons. The slopes in the running sum indicate changes in regimes of seasonal temperature fluctuations. The anticorrelation provides additional support to the results shown in Figs. 9 and 10, which suggest that atmospheric processes in the subtropics and mid-latitudes of the $\mathrm{SH}$ and interactions with the Southern Oceans play an important role to moderate global variations of temperature on decadal time-scales.

We note additionally that the scaling properties (Fig. 12) obtained from New Zealand and United Kingdom are very consistent with those in Fig. 4. The scaling coefficient $H$ estimated from Fig. 11 for United Kingdom is equal to 0.42 and for New Zealand is equal to 0.34 within the 1.0-9.0 years time scale range. Similar $H$ coefficients are observed around New Zealand and Northern Europe (typically at 6.0 7.5 years time-scale) in Fig. 4.

\section{Conclusions}

The anti-persistence of the temperature field on inter-decadal time scales is part of the decadal variability of the climate system and this property has not been identified before. Processes at time scales longer than that of ENSO are also responsible for maintaining stationarity in the temperature anomaly field. In addition, our results indicate the importance of the Southern Oceans in regulating temperature fluctuation regimes on long time-scales. The origin of interdecadal fluctuations in the climate system is currently one of the most challenging problems in climate dynamics with 
known global impacts on climate and oceanic ecosystems (Francis et al., 1998; Mantua et al., 1997). For example, the interdecadal fluctuations of the large-scale circulation over the North Pacific (Deser et al., 2004; Trenberth and Hurrell, 1994) has been hypothesized to occur from a variety of possibilities ranging from: 1) coupled ocean-atmosphere interactions (Latif and Barnett, 2005), 2) air-sea interactions in the subtropical Pacific with atmospheric teleconnections to the North Pacific (Knutson and Manabe, 1998), 3) extratropicaltropical interactions via an equatorward subsurface oceanic pathway and a return atmospheric bridge ( $\mathrm{Gu}$ and Philander, 1997) and 4) climate noise arising from integrating stochastic atmospheric variability over the North Pacific Ocean mixed layer and the thermocline (Pierce et al., 2001; Frankignoul et al., 2000). Interestingly, the variations and time-scale of antipersistence are consistent with the variability of the strength of the wintertime atmospheric circulation over North Pacific (20-30 yrs). As such, the anti-persistence reported here constitutes a new and additional piece in the puzzle of interdecadal oscillations in the climate system.

Acknowledgements. The authors acknowledge the following funding supports: L. M. V. Carvalho and H. R. Rocha FAPESP (Proc. 02/09289-9); L. M. V. Carvalho CNPq (302203/02-8, 474033/04-0); A. A. Tsonis ATM-0438612; P. S. Polito CNPq (477959/2004-0).

Edited by: B. D. Malamud

Reviewed by: three anonymous referees

\section{References}

Alley, R. B., Marotzke, J., Nordhaus, W. D., Overpeck, J. T., Peteet, D. M., Pielke Jr., R. A., Pierrehumbert, R. T., Rhines, P. B., Stocker, T. F., Talley, L. D., and Wallace, J. M.: Abrupt climate change, Science, 299, 2005-2010, 2003.

Baba, K., Minobe, S., Kimura, N., and Wakatsuchi, M.: Intraseasonal variability of sea-ice concentration in the Antarctic with particular emphasis on wind effect, J. Geophys. Res., 111, C12023, doi:10.1029/2005JC003052, 2006.

Buldyrev, S. V., Goldberger, A. L., Havlin, S., Mantegna, R. N., Matsa, M. E., Peng, C.-K., Simons, M., and Stanley, H. E.: Longrange correlation properties of coding and noncoding DNA sequences: GenBank analysis, Phys. Rev. E, 51, 5084-5091, 1995.

Beniston, M.: The 2003 heat wave in Europe: A shape of things to come? An analysis based on Swiss climatological data and model simulations, Geophys. Res. Lett., 31, L02202, doi:10.1029/2003GL018857, 2004.

Briffa, K. R., Jones, P. D., and Schweingruber, F. H.: Summer Temperature Patterns over Europe - a Reconstruction from 1750Ad Based on Maximum Latewood Density Indexes of Conifers, Quaternary Res., 30, 36-52, 1988.

Deser, C., Phillips, A. S., and Hurrell, J. W.: Pacific interdecadal climate variability, Linkages between the tropics and the North Pacific during boreal winter since 1900, J. Climate 17, 31093124, 2004.
Eccles, F. and Tziperman, E.: Nonlinear effects on ENSP's period, J. Atmos. Sci., 61, 474-482, 2004.

Emanuel, K.: Increasing destructiveness of tropical cyclones over the past 30 years, Nature, 436, 686-688, doi:10.1038/nature03906, 2005.

Feder, J.: Fractals, Plenum, New York, 283 pp., 1988.

Francis, R. C., Hare, S. R., Hollowed, A. B., and Wooster, W. S.: Effects of interdecadal climate variability on the oceanic ecosystems of the NE Pacific, Fish. Oceanogr., 7, 1-21, 1998.

Frankignoul, C., Kestenare, E., Sennechael, N., de Coetlogon, G., and D'Andrea, F.: On decadal-scale ocean-atmosphere interactions in the extended ECHAM1/LSG climate simulation, Clim. Dyn., 16, 333-354, 2000.

Gu, D. F. and Philander, S. G. H.: Interdecadal climate fluctuations that depend on exchanges between the tropics and extratropics, Science, 275, 805-807, 1997.

Holton, J.: An introduction to dynamic meteorology, 4th ed., Elsevier, Academic Press, 535 pp., 2004.

Hurst, H. E.: Long-term storage capacity of reservoirs, Trans. Amer. Soc. Civ. Eng., 116, 770-808, 1951.

Hurst, H. E., Black, R. P., and Simaika, Y. M.: Long-term storage: An experimental study, Constable, London, 145 pp., 1965.

IPCC: Climate Change 2007: The Physical Science Basis. Contribution of Working Group I to the Fourth Assessment Report of the Intergovernmental Panel on Climate Change, edited by: Solomon, S., Qin, D., Manning, M., Chen, Z., Marquis, M., Averyt, K. B., Tignor, M., and Miller, H. L., Cambridge University Press, Cambridge, UK, 996 pp., 2007.

Kalnay, E., Kanamitsu, M., Kistler, R. Collins, W., Deaven, D. , Gandin, L., Iredell, M., Saha, S., White, G., Woollen, J., Zhu, Y., Leetmaa, A., Reynolds, B., Chelliah, M. Ebisuzaki, W., Higgins, W., Janowiak, J., Mo, K. C., Ropelewski, C., Wang, J., Jenne, R., and Joseph, D., et al.: The NCEP-NCAR 40 Year Reanalysis Project, B. Am. Meteorol. Soc., 77, 437-471, 1996.

Karl, T. R., Knight, R. W., and Plummer, N.: Trends in HighFrequency Climate Variability in the 20th-Century, Nature, 377, 217-220, 1995.

Karl, T. R. and Knight, R. W.: The 1995 Chicago heat wave: how likely is a recurrence?, B. Am. Meteorol. Soc., 78, 1107-1119, 1997.

Koscielny-Bunde, E., Bunde, A., Havlin, S., Roman, H. E., Goldreich, Y., and Schellnhuber, H.-J.: Indication of a universal persistence law governing atmospheric variability, Phys. Rev. Lett., 81(3), 729-732, 1998.

Knutson, T. R. and Manabe, S.: Model assessment of decadal variability and trends in the tropical Pacific Ocean, J. Climate, 11, 2273-2296, 1998.

Latif, M. and Barnett, T. P.: Decadal climate variability over the North Pacific and North America: Dynamics and predictability, J. Climate, 9, 2407-2423, 1996.

Lau, W. K. M. and Waliser, D. E.: Intraseasonal Variability in the atmosphere-ocean climate system, Springer, New York, 435 pp., 2005.

Madden, R. A. and Julian, P. R.: Detection of a 40-50 day oscillation in the tropics, J. Atmos. Sci., 43, 3138-3158, 1994.

Malamud, B. D. and Turcotte, D. L.: Self-affine time series: I. Generation and analyses, Adv. Geophys., 40, 1-90, 1999.

Mandelbrot, B. B: The fractal geometry of nature, Freeman, New York, 468 pp., 1983. 
Mann, M. E., Bradley, R. S., and Hughes, M. K.: Global-scale temperature patterns and climate forcing over the past six centuries, Nature, 392, 779-787, 1998.

Mantua, N. J., Hare, S. R., Zhang, Y., Wallace, J. M., and Francis, R. C.: A Pacific interdecadal climate oscillation with impacts on salmon production, B. Am. Meteorol. Soc., 78, 1069-1079, 1997.

Maraun, D., Rust, H. W., and Timmer, J.: Tempting long-memory on the interpretation of DFA results, Nonlin. Processes Geophys., 11, 495-503, 2004, http://www.nonlin-processes-geophys.net/11/495/2004/.

Montroll, E. W. and Shlesinger, M. F.: Nonequilibrium Phenomena II: From stochastics to hydrodynamics, North Holland, Amsterdam, 296 pp., 1984.

McPhaden, M. J. and Zhang, D. X.: Slowdown of the meridional overturning circulation in the upper Pacific Ocean, Nature, 415, 603-608, 2002.

Meehl, G. A. and Tebaldi, C.: More intense, more frequent, and longer lasting heat waves in the 21st century, Science, 305, 994997, 2004.

Pierce, D. W., Barnett, T. P., Schneider, N., Saravanan, R., Dommenget, D., and Latif, M.: The role of ocean dynamics in producing decadal climate variability in the North Pacific., Clim. Dynam., 18, 51-70, 2001.

Peitgen, H.-O. and Saupe, D.: The science of fractal images, Springer-Verlag, New York, 312 pp., 1998.

Peixoto, J. P. and Oort, A. H.: Physics of Climate, American Institute of Physics, New York, NY, 520 pp., 1992.

Pelletier, J. D.: Analysis and modeling of the natural variability of the climate, J. Climate, 10, 1331-1342, 1997.
Peng, C.-K., Buldyrev, S. V., Goldberger, A. L., Havlin, S., Sciortino, F., Simons, M., and Stanley, H. E.: Long-range correlations in nucleotide sequences, Nature, 356, 168-170, 1992.

Przybylak, R.: Changes in seasonal and annual high-frequency air temperature variability in the Arctic from 1951 to 1990, Int. J. Climatol, 22, 1017-1032, 2002.

Serreze, M. C., Maslanik, J. A., Scambos, T. A., Fetterer, F., Stroeve, J., Knowles, K., Fowler, C., Drobot, S., Barry, R. G., and Haran, T. M.: A record minimum arctic sea ice extent and area in 2002, Geophys. Res. Lett., 30(3), 1110 , doi:10.1029/2002GL016406, 2003.

Shepherd, A., Wingham, D., Payne, T., and Skvarca, P.: Larsen ice shelf has progressively thinned, Science, 302, 856-859, 2003.

Stanley, H. E.: Introduction to phase transitions and critical phenomena, Oxford University Press, London, 308 pp., 1971.

Trenberth, K. E. and Hurrell, J. W.: Decadal Atmosphere-Ocean Variations in the Pacific, Clim. Dynam., 9, 303-319, 1994.

Tsonis, A. A.: Chaos from theory to applications, Plenum, New York, 274 pp., 1992.

Tsonis, A. A., Roebber, P. J., and Elsner, J. B.: A characteristic time scale in the global temperature record, Geophys. Res. Lett., 25, 2821-2823, 1998

Tsonis, A. A., Hunt, A. G., and Elsner, J. B.: On the relation between ENSO and global climate change, Meteor. Atmos. Phys., 84, 229-242, 2003.

Xiong, L. and Palmer, J. G.: Reconstruction of New Zealand temperatures back to AD 1720 using Libocedrus bidwillii tree-rings, Climatic Change, 45, 339-359, 2000. 